\title{
Biliary intraepithelial neoplasia: an international interobserver agreement study and proposal for diagnostic criteria
}

\author{
Yoh Zen ${ }^{1}$, N Volkan Adsay ${ }^{2}$, Krystof Bardadin ${ }^{3}$, Romano Colombari ${ }^{4}$, Linda Ferrell ${ }^{5}$, \\ Hironori Haga $^{6}$, Seung-Mo Hong ${ }^{7}$, Prodromos Hytiroglou ${ }^{8}$, Günter Klöppel ${ }^{9}$, \\ Gregory Y Lauwers ${ }^{10}$, Dirk J van Leeuwen ${ }^{11}$, Kenji Notohara ${ }^{12}$, Kiyoko Oshima ${ }^{13}$, \\ Alberto Quaglia ${ }^{14}$, Motoko Sasaki ${ }^{1}$, Fausto Sessa ${ }^{15}$, Arief Suriawinata ${ }^{11}$, Wilson Tsui ${ }^{16}$, \\ Yutaka Atomi ${ }^{17}$ and Yasuni Nakanuma ${ }^{1}$
}

${ }^{1}$ Department of Human Pathology, Kanazawa University Graduate School of Medicine, Kanazawa, Japan; ${ }^{2}$ Department of Pathology, Harper Hospital, Wayne State University, Detroit, MI, USA; ${ }^{3}$ Department of Pathology, Medical Centre for Postgraduate Education, Warsaw, Poland; ${ }^{4}$ Department of Pathology, 'G. Fracastoro' City Hospital, Verona, Italy; ${ }^{5}$ Department of Pathology, University of California San Francisco, San Francisco, CA, USA; ${ }^{6}$ Laboratory of Anatomic Pathology, Kyoto University Hospital, Kyoto, Japan; ${ }^{7}$ Department of Pathology, University of Virginia Health System, Charlottesville, VA, USA; ${ }^{8}$ Department of Pathology, Aristotle University Medical School, Thessaloniki, Greece; ${ }^{9}$ Department of Pathology, University of Kiel, Kiel, Germany; ${ }^{10}$ Department of Pathology, Gastrointestinal Pathology Service, Massachusetts General Hospital and Harvard Medical School, Boston, MA, USA; ${ }^{11}$ Section of Gastroenterology/Hepatology and Pathology, Dartmouth Medical School/Dartmouth-Hitchcock Medical Center, Lebanon, NH, USA;

${ }^{12}$ Department of Pathology, Kurashiki Central Hospital, Kurashiki, Japan; ${ }^{13}$ Department of Pathology, Loyola University Medical Center, Maywood, IL, USA; ${ }^{14}$ Institute of Liver Studies, King's College Hospital, London, UK; ${ }^{15}$ Department of Human Morphology, University of Insubria, Varese, Italy; ${ }^{16}$ Department of Pathology, Caritas Medical Center, Kowloon, Hong Kong and ${ }^{17}$ Department of Surgery, Kyorin University School of Medicine, Tokyo, Japan

\begin{abstract}
Cholangiocarcinoma of the intrahepatic and extrahepatic bile ducts develops through a multistep histopathologic sequence. Premalignant or non-invasive neoplastic lesions of bile ducts have been historically called biliary dysplasia or atypical biliary epithelium. To this date, no standard terminology or classification system has been offered for these lesions. In 2005, a conceptual framework and diagnostic criteria for biliary intraepithelial neoplasia (BilIN) were proposed using the livers of patients with hepatolithiasis. We report herein an international interobserver agreement study on the diagnosis of biliary non-invasive neoplastic lesions with the goal to obtain a consensus on the terminology and grading. Seventeen pathologists from the United States, Europe and Asia participated in this study. They shared a digital file containing histological pictures of 30 foci of non-invasive neoplastic lesions selected from the biliary system of patients suffering from primary sclerosing cholangitis, choledochal cyst or hepatolithiasis. In the criteria, we proposed in 2005, BillN was classified into three categories based on the degree of atypia: BillN-1, BillN-2 and BillN-3. In this study, consensus was reached for the terminology of BilIN and the three-grade classification system. Interobserver agreement on the diagnosis was moderate $(\kappa$-value $=0.45)$. On the basis of the suggestions and opinions obtained from the 17 participants, the original criteria for BillN were revised. We now propose a new consensus classification of BillN that may assist in allowing a more uniform terminology for the diagnosis of biliary noninvasive neoplastic lesions. This classification should help to advance clinical and research applications. Modern Pathology (2007) 20, 701-709; doi:10.1038/modpathol.3800788; published online 13 April 2007
\end{abstract}

Keywords: biliary dysplasia; BillN; intrahepatic cholangiocarcinoma; cholangiocarcinoma; bile duct

Correspondence: Dr Y Nakanuma, MD, Department of Human Pathology, Kanazawa University Graduate School of Medicine, 13-1 Takaramachi, Kanazawa 920-8640, Japan.

E-mail: pbcpsc@kenroku.kanazawa-u.ac.jp

Received 7 December 2006; revised 12 March 2007; accepted 15 March 2007; published online 13 April 2007
Specialties such as gynecology, urology and gastroenterology are facing difficult dilemmas when trying to incorporate 'preneoplastic lesions' into management recommendations that may vary from observa- 
tion only to more radical surgical approaches. ${ }^{1-3}$ Interobserver agreement studies have been introduced as a way to enhance consensus on terminology and classification of lesions. ${ }^{4-8}$ The study of pathogenesis and disease outcome would be facilitated by classification system that is devoid of subjective interpretation and could uniformly be applied worldwide, thus enabling clinicians and scientists to share and compare information.

Intrahepatic and extrahepatic cholangiocarcinoma develop through a multistep carcinogenesis, and are preceded by premalignant or in situ neoplastic lesions. ${ }^{9,10}$ At least two major precursor lesions have been associated with the development of invasive cholangiocarcinoma. The first is a microscopic lesion of flat or low-papillary dysplastic epithelium, and known by names, such as biliary dysplasia, atypical biliary epithelium or carcinoma in situ. ${ }^{9}$ The second is an intraductal papillary neoplasm of the bile duct (biliary IPN), which is a macroscopic lesion characterized by prominent papillary proliferation of dysplastic epithelium with frequent intestinal metaplasia and mucin hypersecretion. ${ }^{11-14}$ Biliary IPN is now regarded as the biliary counterpart of pancreatic intraductal papillary mucinous neoplasm. ${ }^{15-17}$ These two lesions are different in their pathological and clinical characteristics. ${ }^{11,17}$ However, both of them may occur in the bile duct of patients suffering from primary sclerosing cholangitis (PSC), choledochal cyst or hepatolithiasis, all of which are known to predispose for the development of cholangiocarcinoma. ${ }^{11-13,15}$

In 2005, we conducted an interobserver and intraobserver agreement study on the diagnosis of biliary dysplasia of the bile ducts affected by hepatolithiasis, and proposed a tentative set diagnostic criteria. ${ }^{18}$ We used the term 'biliary intraepithelial neoplasia' (BilIN) instead of biliary dysplasia, according to the World Health Organization classification of tumors. ${ }^{19}$ BillN was classified into three categories (grades) based on the degree of cellular and structural atypia: BilIN-1, BilIN-2 and BilIN-3. These diagnostic criteria have been subject of criticism. For example, the proposal of BiliN in this previous study was based solely on the intrahepatic biliary lesions of patients with hepatolithiasis. It remained unclear if those diagnostic criteria would also apply to premalignant lesions of the extrahepatic bile ducts or those occurring in other diseases such as PSC and choledochal cyst. Another criticism was that all pathologists enrolled in the previous study were from Asian countries (Japan and Korea). ${ }^{18}$

We now report on the results of an international interobserver agreement study of the histological diagnosis of biliary dysplastic lesions conducted among 17 pathologists from the United States and Europe, in addition to Asia. This study did not include cases of biliary IPN. The aim of this study was to establish international histological criteria of BilIN, which could be applied to biliary non- invasive neoplastic lesions in the intrahepatic and extrahepatic bile ducts of various biliary diseases.

\section{Materials and methods}

\section{Case Selection}

Histological specimens were selected from the resected or explanted material of patients with PSC $(n=6)$, choledochal cyst $(n=7)$ and hepatolithiasis $(n=10)$. The male to female ratios and average ages were as follows: PSC, $4: 2$ and 58.2 years (36-67 years); choledochal cyst, 3:4 and 25.9 years (18-35 years); hepatolithiasis, 3:7 and 53.7 years (range: 46-68 years). None of the cases was associated with an invasive cholangiocarcinoma. From these specimens, we selected a total of 30 lesions ranging from reactive to dysplastic biliary epithelium (10 lesions from each disease). The lesions from the PSC and hepatolithiasis patients were from large intrahepatic or hilar bile ducts, whereas those from choledochal cyst were of extrahepatic bile duct. No gallbladder lesions were included in this study. The lesions were not macroscopically detectable and did not histologically correspond to biliary IPN. Two digital images (original magnification; $\times 100$ and $\times 400$ ) of each lesion were taken at 5600000 pixels using a microscopic digital camera (DP50, Olympus Corporation, Tokyo, Japan). They were randomly arranged in a single file and numbered from case 1-30.

\section{Terminology of Biliary Epithelial Lesions}

The term 'biliary dysplastic lesion' has been commonly used to describe non-papillary premalignant lesions or in situ neoplastic lesions of the bile duct. In this study, we used the term 'BilIN', because this terminology was used in the World Health Organization classification of tumors, in a similar fashion as for premalignant lesions of other organs such as pancreas (pancreatic intraepithelial neoplasia $(\mathrm{Pa}-$ nIN)) and prostate (prostatic intraepithelial neoplasia (PIN)). ${ }^{20,21}$ In the biliary system, three grading systems have been commonly used. Some groups classify these lesions as mild, moderate or severe dysplasia. Other groups use low- and high-grade dysplasia and carcinoma in situ. In this study, noninvasive dysplastic lesions of biliary epithelium were classified into three histological grades; BilIN-1, BilIN-2 and BilIN-3. BilIN-3 includes so-called 'carcinoma in situ'. The terminology and classification are in line with the current concept suggesting that the spectrum of these lesions reflects a multistep carcinogenic sequence with increasing neoplastic potential.

\section{Study Design}

The file containing the digital images of the 30 lesions was sent to a panel of 17 pathologists with 
expertise in hepatobiliary pathology. Only the digital file was available for diagnostic purposes. The clinical data and underlying conditions were not disclosed. The study participants were asked to categorize each lesion as reactive change, BilIN-1, BilIN-2 or BilIN-3, applying the tentative criteria reported previously. ${ }^{18}$ Briefly, BilIN-1 showed mild cellular/nuclear atypia such as nuclear membrane irregularity or nuclear enlargement with only a minimal disturbance of cellular polarity. BilIN-2 had evident cellular/nuclear atypia, but not enough to suggest overt carcinoma, with a focal disturbance of cellular polarity. BilIN-3 showed a diffuse disturbance of cellular polarity with or without distinct cellular/nuclear atypia corresponding to an overt carcinoma. Next, the participants were asked whether they could determine the underlying disease (hepatolithiasis, PSC or choledochal cyst) based on the nature of the epithelial changes of BilIN alone. Finally, they were asked to provide suggestions and modifications of the diagnostic criteria. Two of the authors (YN and YZ) performed the data analysis. The initial diagnostic criteria for BilIN were modified based on the suggestions. We subsequently obtain a consensus on the new classification criteria for BilIN.

\section{Pathologists Enrolled}

Seventeen pathologists participated in this study as participants and are all co-author of this article. Yasuni Nakanuma, Yoh Zen and Yutaka Atomi, who selected the specimens and analyzed the data, did not participate in the diagnostic evaluation. No pathologists except one (MS) participated in the previous interobserver agreement study. ${ }^{18}$ Two pathologists (DvL and AS) jointly diagnosed each lesion as a single participant. Therefore, the interobserver agreement study consisted of 17 pathologists and 16 participants. The pathologists enrolled in this study specialize in digestive system, particularly the hepatobiliary and pancreatic system.

\section{Data Analysis}

Interobserver agreement was evaluated according to the concordant rate (\%) and the $\kappa$-value. Interpretations for the $\kappa$-value were as described previously, ${ }^{22}$ that is, $<0.00$, poor agreement; $0.00-0.20$, slight agreement; 0.21-0.40, fair agreement; 0.41-0.60, moderate agreement; $0.61-0.80$, substantial agreement; and 0.81-1.00, almost perfect agreement.

\section{Results of the Interobserver Agreement Study}

Among the 30 lesions assessed, the highest level of agreement was reached with respect to the diagnosis: reactive changes in six lesions, BilIN-1 in seven lesions, BilIN-2 in 13 lesions and BilIN-3 in 4 lesions. More than 9 of 16 participants agreed with the diagnosis of 21 of 30 lesions. Twelve of those lesions are shown in Figures 1-4. Similarly, a consensus of more than 11 participants was obtained for 12 lesions. Especially, almost perfect consensus was reached in two lesions in which 15 out of 16 participants diagnosed BilIN-3. The concordant rate and $\kappa$-value for the diagnosis of 30 lesions were 60.3 and $0.45 \%$, respectively. $\kappa$-value for each lesion was 0.42 for reactive change, 0.40 for BilIN-1, 0.16 for BilIN-2 and 0.44 for BilIN-3 (Table 1). Then these participants were categorized into three groups based on their countries: the United States (five participants; NVA, LF, GYL, DvL KO and AS), Europe (six participants; KB, RC, PH, GK, AQ and FS) and Asia (five participants; $\mathrm{HH}, \mathrm{SMH}, \mathrm{KN}, \mathrm{MS}$ and WT). There was no suggestion that were related to regional differences with respect to the diagnosis. As shown in Table 1, no significant difference was either observed in $\kappa$-values for the diagnosis of biliary lesions among three groups.

None of the participants could identify the preceding condition solely based on epithelial changes of BilIN, although seven participants suggested background disease from histological changes of the bile duct wall, such as lamellar fibrosis, inflammation and stromal edema in some cases. Interestingly, one participant suggested that BilIN could be clustered into six groups based on the patterns of epithelial changes themselves: (i) pseudopapillary eosinophilic epithelium with tufting and atypia (eight lesions; Figure 4a and c); (ii) long thin papilla (one lesion); (iii) short thick papilla (three lesions; Figures 3a and 4b); (iv) adenoma-like with glandular involvement (four lesions; Figure 3c); (v) micropapillary with/without intestinal metaplasia (eight lesions; Figures 2a, c and 3b); (vi) others (six lesions including reactive change). This participant did not describe the relationship between those histological groups and preceding conditions. However, it was found that seven out of eight lesions of (i) pseudopapillary eosinophilic epithelium with tufting and atypia arose in hepatolithiasis (three cases) and choledochal cyst (four cases). Three lesions of (iii) short thick papilla were all hepatolithiasis cases. (iv) Adenoma-like with glandular involvement mainly consisted of PSC cases (three out of four cases). Similarly, most lesions of (v) micropapillary with/without intestinal metaplasia derived from PSC (four cases) and choledochal cyst (three cases). That is, BilIN occurring in hepatolithiasis frequently showed (i) pseudopapillary eosinophilic epithelium with tufting and atypia or (iii) short thick papilla. BilIN of PSC commonly manifested (iv) adenoma-like with glandular involvement or (v) micropapillary with/ without intestinal metaplasia. BilIN of choledochal cyst often showed (i) pseudopapillary eosinophilic epithelium with tufting and atypia or (v) micropapillary with/without intestinal metaplasia. 

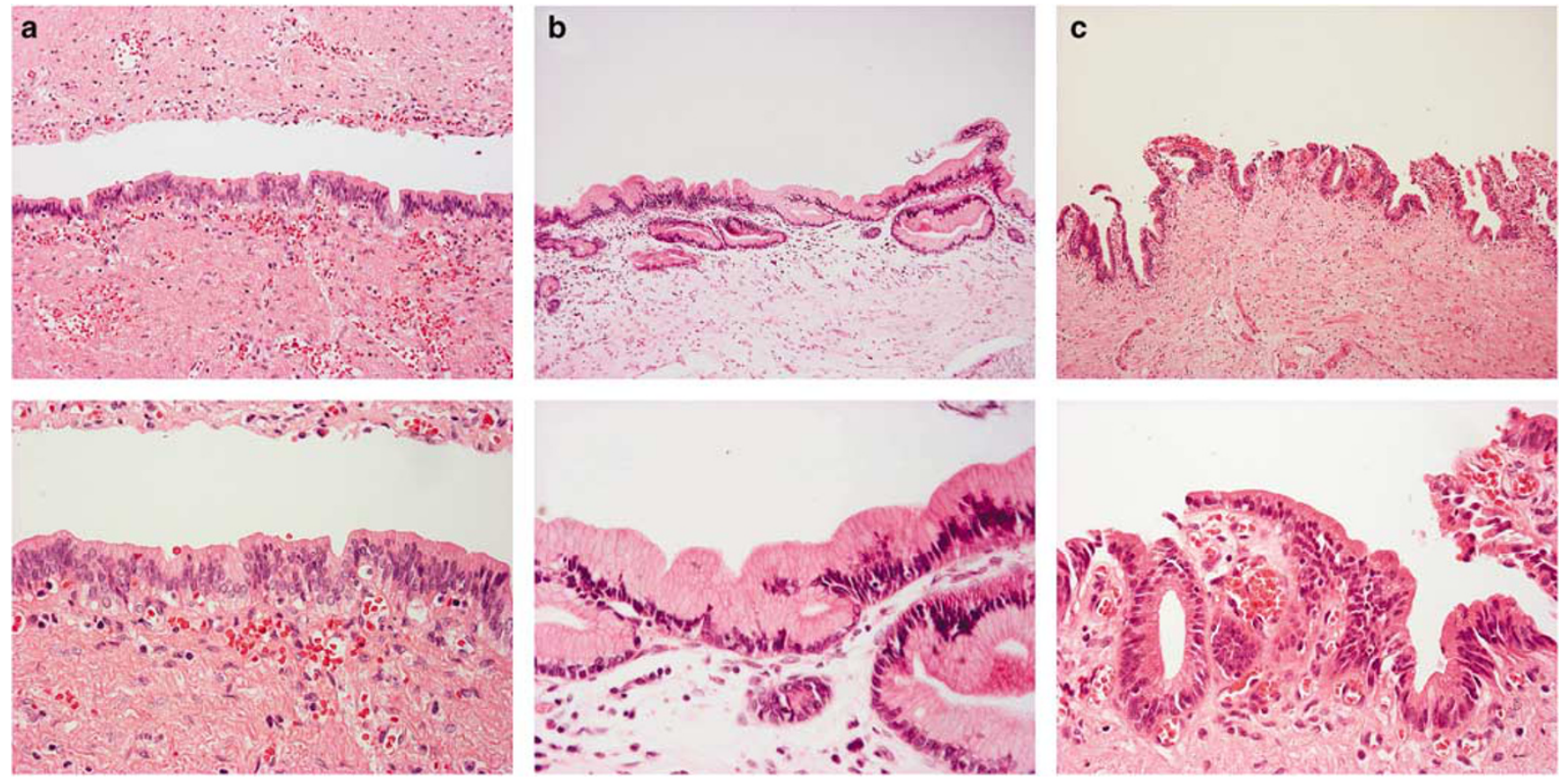

Figure 1 Histological pictures of biliary epithelia diagnosed as reactive changes by more than nine participants (hematoxylin and eosin; upper, $\times 100$; lower, $\times 400)$.
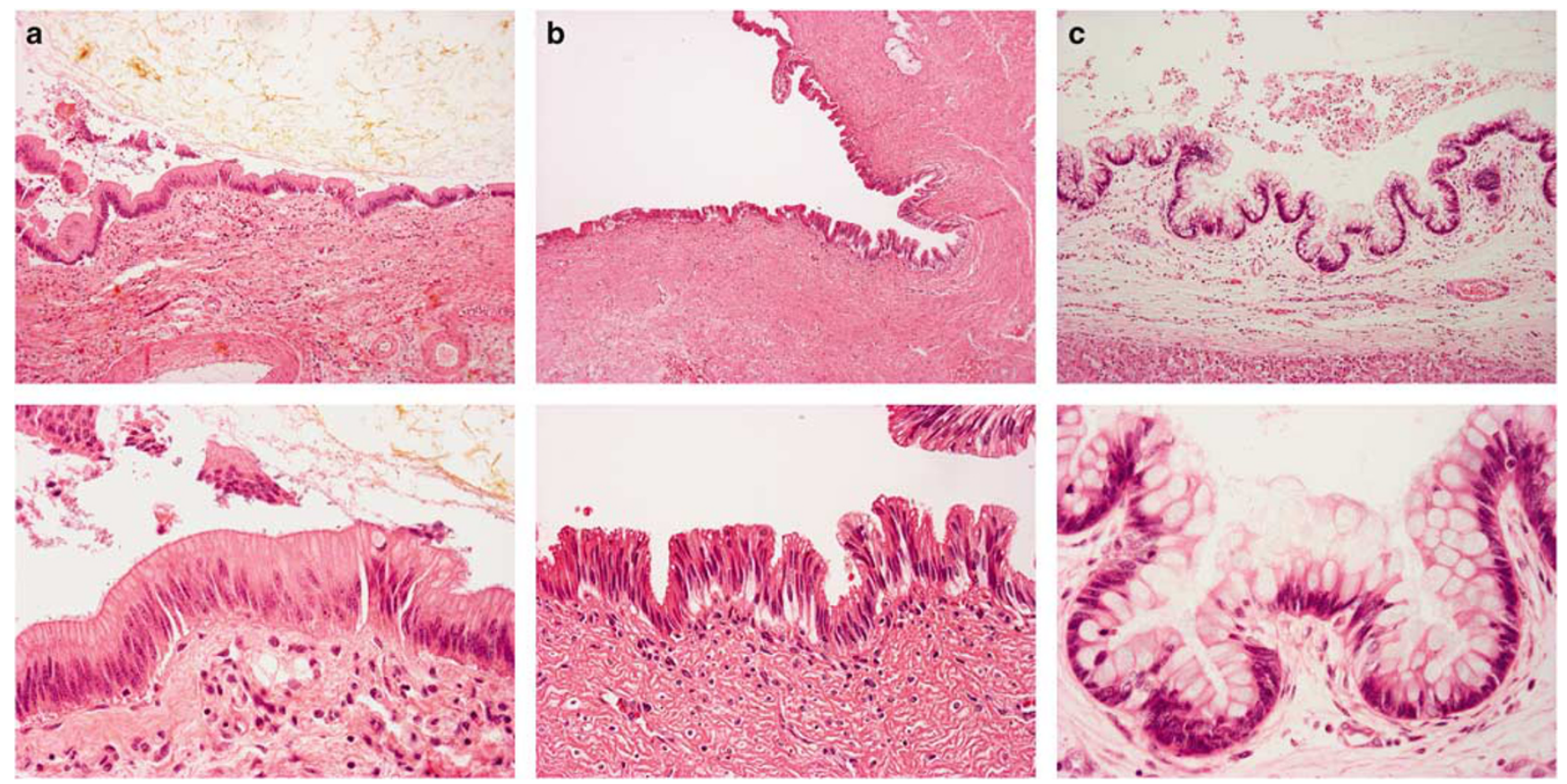

Figure 2 Histological pictures of biliary lesions diagnosed as BilIN-1 by more than nine participants (hematoxylin and eosin; upper, $\times 100$; lower, $\times 400)$.

\section{Proposal of Histological Criteria for BilIN}

All participants agreed on the basic concept of the diagnostic criteria, including the terminology (BilIN) and their classification (BilIN-1, -2 and -3). In addition, several suggestions and opinions for the modification of diagnostic criteria were obtained. In the tentative criteria, micropapillary and non-papillary lesions were separately described in each lesion. In the present study, most participants mentioned that each lesion should be defined irrespective of their architectures because papillary 

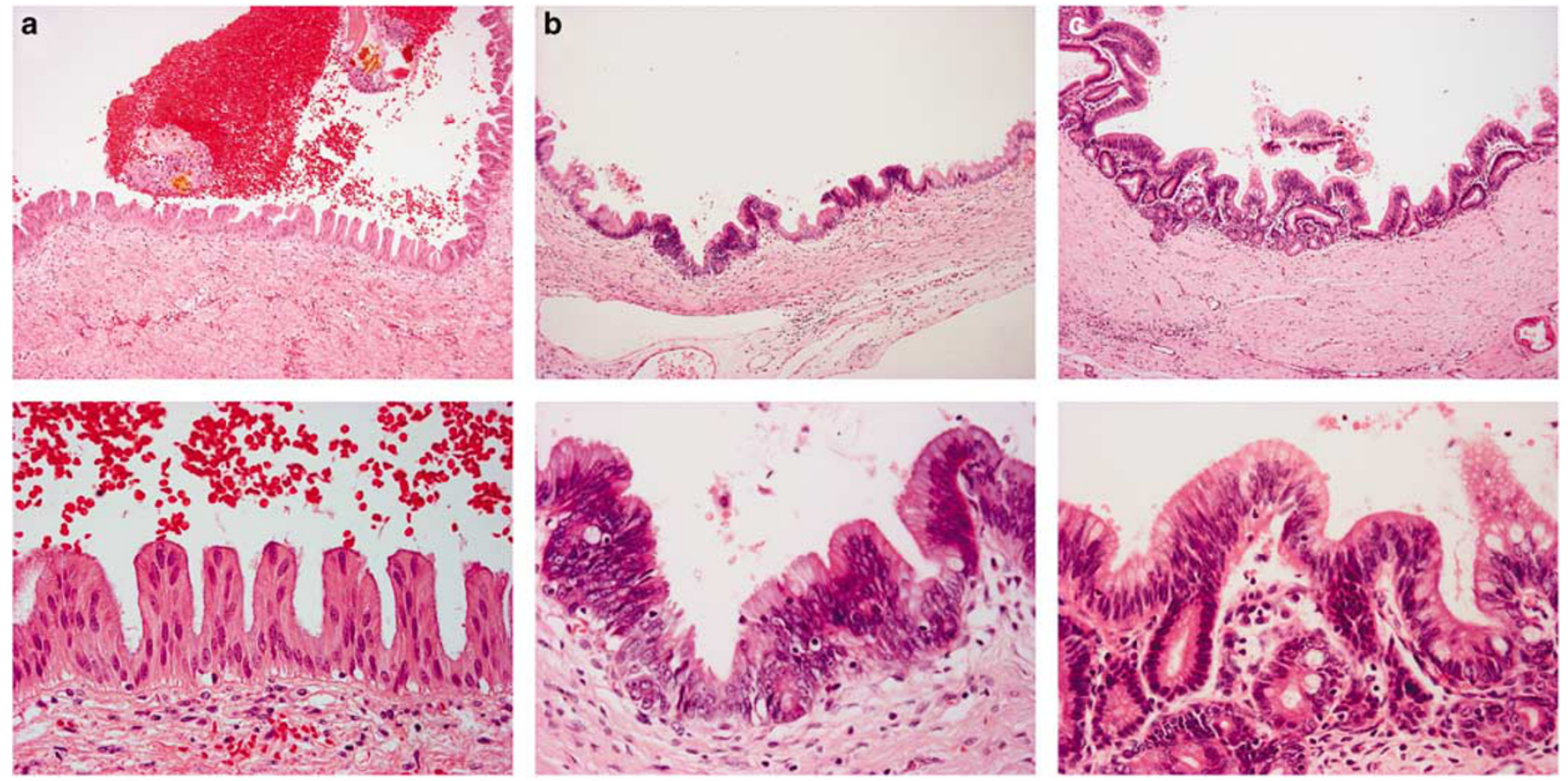

Figure 3 Histological pictures of biliary lesions diagnosed as BilIN-2 by more than nine participants (hematoxylin and eosin; upper, $\times 100$; lower, $\times 400$ ).
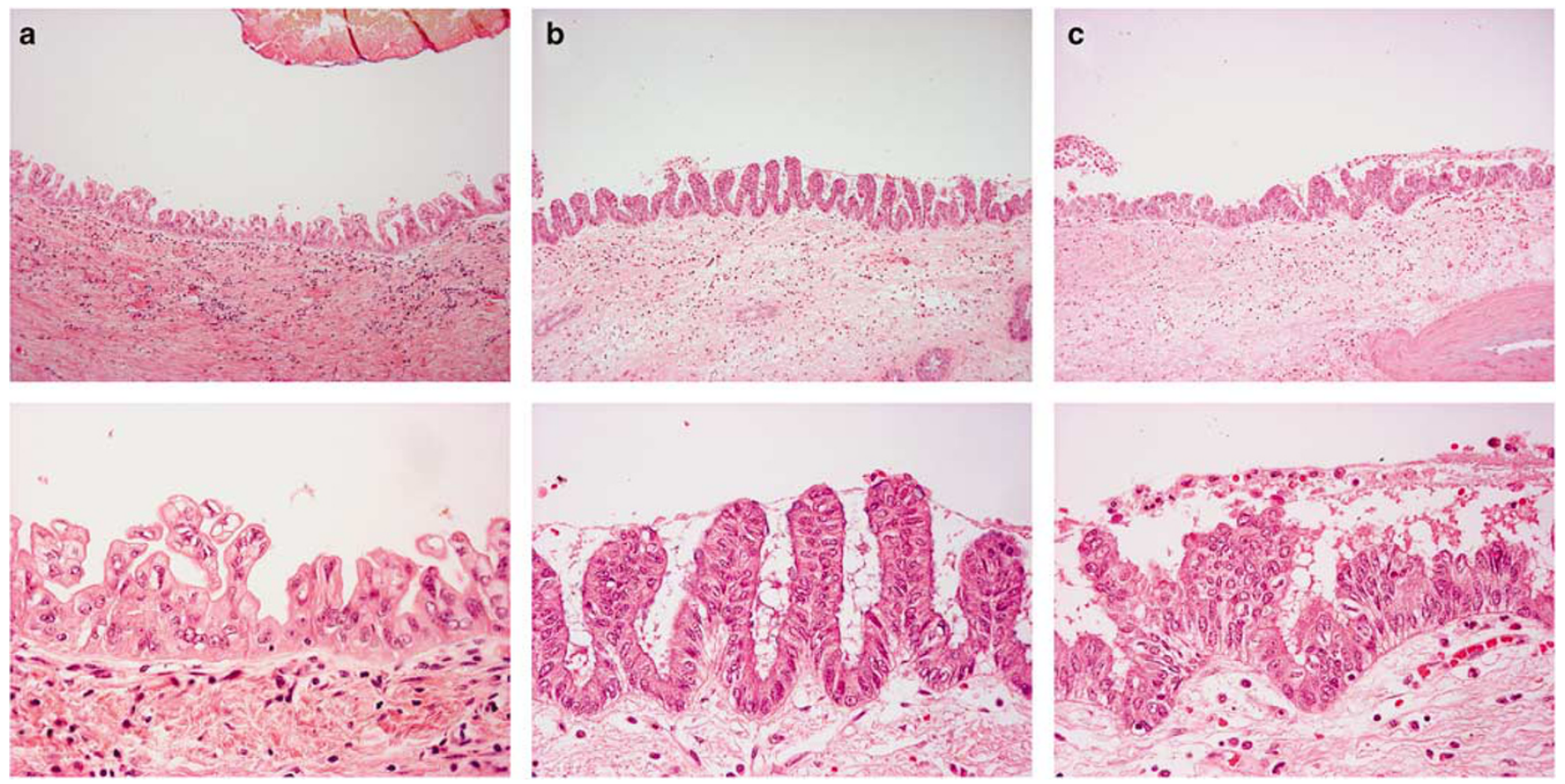

Figure 4 Histological pictures of biliary lesions diagnosed as BilIN-3 by more than nine participants (hematoxylin and eosin; upper, $\times 100$; lower, $\times 400$ ).

lesions are rare in BilIN, and the term 'papillary' might cause confusion with the other papillary premalignant lesion (biliary IPN).

The next suggestion was about description of cellular and nuclear atypia. Detailed descriptions about cellular and nuclear atypia might assist many pathologists, particularly general pathologists, to promote a more general use of this classification. It was also suggested that mitosis in BilIN is one of the features indicating a diagnosis of BilIN-2 or BilIN-3. Intraepithelial neutrophils in the reactive epithelium might be a feature to differentiate it from BilIN-1. Taking these suggestions into consideration, we modified the criteria and establish the consensus 
Table 1 Kappa values of interobserver agreement on the diagnosis of reactive and dysplastic biliary epithelial lesions occurring in primary sclerosing cholangitis, choledochal cyst and hepatolithiasis

\begin{tabular}{|c|c|c|c|c|c|c|}
\hline & $\mathrm{N}$ & Reactive & BilIN-1 & BilIN-2 & BilIN-3 & Total \\
\hline United States & 5 & 0.43 & 0.40 & 0.19 & 0.43 & 0.47 \\
\hline Europe & 6 & 0.40 & 0.41 & 0.12 & 0.43 & 0.44 \\
\hline Asia & 5 & 0.44 & 0.40 & 0.16 & 0.45 & 0.46 \\
\hline Total & 16 & 0.42 & 0.40 & 0.16 & 0.44 & 0.45 \\
\hline
\end{tabular}

$\mathrm{N}$, number of participants.

Table 2 Modified diagnostic criteria for reactive change of the biliary epithelium and biliary intraepithelial neoplasia

Hyperplasia or regenerative change

These lesions are most commonly flat. Low-papillary or micropapillary architecture, when observed, are often associated with hepatolithiasis or choledochal cyst, but tall papillary structures are rare. Cellularity is only slightly increased compared to normal biliary epithelium. Nuclei are round or oval, and slightly enlarged with smooth nuclear membrane. Chromatin is fine and homogeneously distributed. Intraepithelial neutrophil infiltration can be seen. Mitoses can be observed in some lesions. ${ }^{a}$

BilIN-1 (biliary intraepithelial neoplasia-1)

These lesions show flat or micropapillary architecture. Nuclei are basally located. Some lesions show focal nuclear pseudostratification; however, the nuclei remain within the lower two thirds of the epithelium. Cytologically, mild nuclear abnormalities, such as subtle irregularities of nuclear membrane, high nuclear/cytoplasmic ratios and nuclear elongation are seen. Nuclear sizes and shapes are relatively uniform, and the presence of large nuclei suggest a diagnosis of BilIN-2 or BilIN-3.

BIIN-2 (biliary intraepithelial neoplasia-2)

These lesions show flat, pseudopapillary or micropapillary architecture. Loss of cellular polarity is easily found, but it is not a diffuse feature. Nuclear pseudostratification reaching the luminal surface is common. Cytologically, dysplastic nuclear changes, which include enlargement, hyperchromasia and irregular nuclear membrane, are evident. Some variations in nuclear sizes and shapes are seen. Peribiliary glands are sometimes involved (glandular involvement). Mitoses are rare.

BilIN-3 (biliary intraepithelial neoplasia-3)

These lesions usually show pseudopapillary or micropapillary architecture, and are only rarely flat. They cytologically resemble carcinoma, but invasion through the basement membrane is absent. Cellular polarity is diffusely and severely distorted with nuclei reaching and piling on the luminal surface. 'Budding off' of small clusters of epithelial cells into the lumen and cribriforming can be seen. Cytologically malignant features with severe nuclear membrane irregularities, hyperchromasia or abnormally large nuclei are typically noted. Mitoses can be observed. Peribiliary gland involvement is sometimes found.

${ }^{\mathrm{a}}$ When present, discrimination from BilIN is important, because mitoses in BilIN suggest the diagnosis of higher grade lesions, mostly BilIN-3.

classification system of BilIN, as shown in Table 2. We defined each lesion irrespective of architecture and for each described the detailed cellular and nuclear changes. We also added descriptions about glandular involvement, mitosis, nuclear location and intraepithelial neutrophils in the appropriate categories.

Additional opinions were provided by several participants. One participant mentioned that the two-grade system (low-grade BilIN and high-grade BilIN) might be easier to use than the three-grade system. However, we sometimes found dysplastic lesions difficult to classify simply into low or high grade. BilIN might be biologically divided into two categories (indolent or progressive), although an intermediate grade should be left until the morphological-biological correlation is elucidated. Two participants suggested that immunostaining could improve this classification. Molecule(s) related to the cell cycle or carcinogenesis, such as p53, p21, p16 and MIB-1, might be candidates for such use. ${ }^{23-25}$ The expressions of most of these molecules is gradually altered during multistep carcinogenesis, and their expression patterns overlap the grades of
BilIN. Therefore, the histological criteria should only be based on morphological features at this time. However, the use of immunostaining may be helpful for BilIN classification and grading, and further studies are necessary to resolve this issue.

In practice, separating reactive inflammatory changes from truly neoplastic lesions is occasionally difficult, especially when the patients have been stented before surgery or biopsy. When in doubt, pathologists are advised to use the terminology of 'indefinite for dysplasia'. However, one should try to avoid this practice as much as possible. In difficult cases, submission and review of additional sections and consultation with an expert pathologist can help solve diagnostic dilemma.

\section{Discussion}

In this study, we examined the interobserver agreement on the diagnosis of BilIN occurring as a complication of PSC, choledochal cyst and hepatolithiasis to obtain a consensus about the terminology and diagnostic criteria for those lesions. We used the 
diagnostic criteria of a previous study, as tentative guidelines. ${ }^{18}$ All pathologists enrolled in the present effort agreed on the basic concept of these criteria, including the terminology and three-grade classification. After the interobserver agreement study, we modified the criteria based on the suggestions and opinions obtained from the 17 pathologists. The major alteration concerns the description of the histological features in each lesion. There were short descriptions about cellular atypia and polarity in the tentative criteria; therefore, we added more detailed descriptions of the architecture, as well as the nuclear and cellular changes of each lesion. In addition, we deleted the subcategories of micropapillary and non-papillary lesions to avoid confusion about the discrimination between BilIN and biliary IPN.

The present interobserver agreement study revealed moderate agreement $(60.3 \%$ and $\kappa=0.45)$ on the diagnosis of biliary lesions based on the common diagnostic criteria. This agreement is similar to that of our previous study, and to those of studies concerning premalignant lesions of other organs. ${ }^{4,6,18,26}$ The results somewhat encouraging since it was the first time for most participants to use these criteria. Furthermore, biliary neoplasms are not as commonly encountered and diagnosed compared to entities such as Barrett esophagus in the West or gastric dysplasia in the East, and thus the lack of exposure may also affect the results. The routine usage of the proposed criteria could improve diagnostic agreement.

Interestingly, the interobserver agreement of BilIN-2 was lower than those of reactive change, BilIN-1 and BilIN-3. This resembles the results obtained from the interobserver agreement study on PanIN. ${ }^{26}$ Hruban et $a l^{26}$ reported that the $\kappa$-values for the diagnosis of PanIN-1, PanIN-2 and PanIN-3 were $0.43,0.14$ and 0.42 , respectively. Intermediate groups of three-grade systems might be characterized by low levels of agreement. If we use the twograde system (low or high grade) in the BilIN classification, this issue might be resolved. However, we often encounter dysplastic lesions that are difficult to determine as low grade or high grade. We thought that the three-grade system seems appropriate at this time, although admittedly the clinical significance with regard to management, if a diagnosis was to be made on biopsy, was not evaluated in this study.

We used cases of PSC, choledochal cyst and hepatolithiasis in this study. All participants diagnosed each lesion without difficulties. It is suggested that the identical diagnostic criteria could be used for BilIN irrespective of the preceding biliary diseases. Similarly, intrahepatic and extrahepatic lesions could be diagnosed based on the common criteria. Although none of the participants could determine the background conditions, one suggested that BilIN might be clustered into six groups based on microscopic features of the biliary epithe- lium. Interestingly, there were some correlations between those groups and the preceding diseases. The histological features of each group might reflect different genetic and epigenetic alterations during cholangiocarcinogenesis in PSC, choledochal cyst and hepatolithiasis. Further analyses employing more cases of BilIN including some from diverse biliary diseases are mandatory to verify this hypothesis.

The histological criteria for BilIN obtained in the present study were based on lesions of intrahepatic and extrahepatic bile ducts. It is well known that similar non-invasive neoplastic lesions can occur in the gallbladder and also in the peribiliary gland. We suggest that the applicability of BilIN classification should be tested for gallbladder and peribiliarygland lesions, because any consensus criteria have not been established for those lesions.

The relationship between BilIN and PanIN is an interesting question. We speculate that BilIN could be seen as the biliary counterpart of PanIN. In previous studies, we showed that BilIN and PanIN exhibited similar expression patterns of mucin core proteins (MUC1 and MUC2), suggesting that identical phenotypic changes occur in both BilIN and PanIN. ${ }^{11,27}$ However, it has not been well documented whether similar genetic alterations also occur in BilIN and PanIN lesions. Thus, further evaluation of oncogenes and tumor-suppressor genes in BilIN and PanIN are necessary to resolve this issue.

Other premalignant lesions, except for BilIN, may occur in the intrahepatic and extrahepatic bile ducts. Biliary IPN is now believed to be the biliary counterpart of pancreatic intraductal papillary mucinous neoplasm. ${ }^{15-17,28-30}$ The discrimination of BilIN and biliary IPN is usually based on the size of the lesion and the proliferation patterns. BiliN is a microscopic lesion and is not grossly identifiable. BilIN microscopically manifests as flat, pseudopapillary (loss of cellular polarity and pseudostratification) and micropapillary lesions (papillary growth with/without indistinct fibrovascular cores). In contrast, biliary IPN is grossly visible, and microscopically characterized by prominent papillary proliferation with distinct fibrovascular cores. The clinicopathological features are different between BilIN and biliary IPN; therefore, this discrimination seems important. ${ }^{11,17}$ However, we rarely encounter premalignant lesions that are as difficult to determine as BilIN vs intraductal spreading of biliary IPN are. It was, therefore, suggested that a consensuses should be obtained in the near future about the discrimination of BilIN and biliary IPN, and the histological grading system of biliary IPN.

In conclusion, this international interobserver agreement study showed that the BilIN system was applicable to premalignant or non-invasive neoplastic lesions of the bile duct identified in the affected bile ducts of PSC, choledochal cyst and hepatolithiasis. We propose a series of revised diagnostic criteria for BilIN. This classification system could 
serve as 'working formulation' and will help the terminology and diagnostic criteria of non-invasive neoplastic lesions of the bile ducts in clinical and research fields.

\section{Acknowledgement}

This study was supported by grants-in-aid for Intrahepatic Calculi from the Ministry of Health and Welfare, Japan.

\section{References}

1 Brentnall TA, Bronner MP, Byrd DR, et al. Early diagnosis and treatment of pancreatic dysplasia in patients with a family history of pancreatic cancer. Ann Intern Med 1999;131:247-255.

2 Canto MI, Goggins M, Yeo CJ, et al. Screening for pancreatic neoplasia in high-risk individuals: an EUSbased approach. Clin Gastroenterol Hepatol 2004;2: 606-621.

3 Bodner K, Bodner-Adler B, Wierrani F, et al. Cold-knife conization versus photodynamic therapy with topical 5-aminolevulinic acid (5-ALA) in cervical intraepithelial neoplasia (CIN) II with associated human papillomavirus infection: a comparison of preliminary results. Anticancer Res 2003;23:1785-1788.

4 Epstein JI, Grignon DJ, Humphrey PA, et al. Interobserver reproducibility in the diagnosis of prostatic intraepithelial neoplasia. Am J Surg Pathol 1995;19: 873-886.

5 Klaes R, Benner A, Friedrich T, et al. p16INK4a immunohistochemistry improves interobserver agreement in the diagnosis of cervical intraepithelial neoplasia. Am J Surg Pathol 2002;26:1389-1399.

6 Bergeron C, Nogales FF, Masseroli M, et al. A multicentric European study testing the reproducibility of the WHO classification of endometrial hyperplasia with a proposal of a simplified working classification for biopsy and curettage specimens. Am J Surg Pathol 1999;23:1102-1108.

7 Longnecker DS, Adsay NV, Fernandez-del Castillo C, et al. Histopathological diagnosis of pancreatic intraepithelial neoplasia and intraductal papillary-mucinous neoplasms: interobserver agreement. Pancreas 2005;31: 344-349.

8 Adsay NV, Basturk O, Bonnett M, et al. A proposal for a new and more practical grading scheme for pancreatic ductal adenocarcinoma. Am J Surg Pathol 2005;29: 724-733.

9 Nakanuma Y, Harada K, Ishikawa A, et al. Anatomic and molecular pathology of intrahepatic cholangiocarcinoma. J Hepatobiliary Pancreat Surg 2003;10: 265-281.

10 Fleming KA, Boberg KM, Glaumann H, et al. Biliary dysplasia as a marker of cholangiocarcinoma in primary sclerosing cholangitis. J Hepatol 2001;34: 360-365.

11 Zen Y, Sasaki M, Fujii T, et al. Different expression patterns of mucin core proteins and cytokeratins during intrahepatic cholangiocarcinogenesis from biliary intraepithelial neoplasia and intraductal papillary neoplasm of the bile duct-an immunohistochemical study of 110 cases of hepatolithiasis. J Hepatol 2006; 44:350-358.

12 Bu-Ghanim M, Suriawinata A, Killackey M, et al. Invasive colloid carcinoma arising from intraductal papillary neoplasm in a 50-year-old woman with primary sclerosing cholangitis. Semin Liver Dis 2004; 24:209-213.

13 Abraham SC, Lee JH, Hruban RH, et al. Molecular and immunohistochemical analysis of intraductal papillary neoplasms of the biliary tract. Hum Pathol 2003; 34:902-910.

14 Kim YS, Myung SJ, Kim SY, et al. Biliary papillomatosis: clinical, cholangiographic and cholangioscopic findings. Endoscopy 1998;30:763-767.

15 Chen TC, Nakanuma Y, Zen Y, et al. Intraductal papillary neoplasia of the liver associated with hepatolithiasis. Hepatology 2001;34:651-658.

16 Kloppel G, Kosmahl M. Is the intraductal papillary mucinous neoplasia of the biliary tract a counterpart of pancreatic papillary mucinous neoplasm? J Hepatol 2006;44:249-250.

17 Zen Y, Fujii T, Itatsu K, et al. Biliary papillary tumors share pathological features with intraductal papillary mucinous neoplasm of the pancreas. Hepatology 2006;44:1333-1343.

18 Zen Y, Aishima S, Ajioka Y, et al. Proposal of histological criteria for intraepithelial atypical/proliferative biliary epithelial lesions of the bile duct in hepatolithiasis with respect to cholangiocarcinoma: preliminary report based on interobserver agreement. Pathol Int 2005;55:180-188.

19 Nakanuma Y, Sripa B, Vantanasapt V, et al. Intrahepatic cholangiocarcinoma. In: Hamilton SR, Aaltonen LA (eds). World Health Organization Classification of Tumours Pathology and Genetics of Tumours of the Digestive System. IARC Press: Lyon, 2000, pp 173-180.

20 Hruban RH, Takaori K, Klimstra DS, et al. An illustrated consensus on the classification of pancreatic intraepithelial neoplasia and intraductal papillary mucinous neoplasms. Am J Surg Pathol 2004;28: 977-987.

21 Epstein JI. Pathology of prostatic intraepithelial neoplasia and adenocarcinoma of the prostate: prognostic influences of stage, tumor volume, grade, and margins of resection. Semin Oncol 1994;21: 527-541.

22 Landis JR, Koch GG. The measurement of observer agreement for categorical data. Biometrics 1977;33: 159-174.

23 Kang YK, Kim WH, Jang JJ. Expression of G1-S modulators (p53, p16, p27, cyclin D1, Rb) and Smad4/Dpc4 in intrahepatic cholangiocarcinoma. Hum Pathol 2002;33:877-883.

24 Bergquist A, Glaumann $\mathrm{H}$, Stal $\mathrm{P}$, et al. Biliary dysplasia, cell proliferation and nuclear DNA-fragmentation in primary sclerosing cholangitis with and without cholangiocarcinoma. J Intern Med 2001;249: 69-75.

25 Lee CS. Ras p21 protein immunoreactivity and its relationship to p53 expression and prognosis in gallbladder and extrahepatic biliary carcinoma. Eur J Surg Oncol 1997;23:233-237.

26 Hruban RH, Adsay NV, Albores-Saavedra J, et al. Pancreatic intraepithelial neoplasia: a new nomenclature and classification system for pancreatic duct lesions. Am J Surg Pathol 2001;25:579-586. 
27 Adsay NV, Merati K, Andea A, et al. The dichotomy in the preinvasive neoplasia to invasive carcinoma sequence in the pancreas: differential expression of MUC1 and MUC2 supports the existence of two separate pathways of carcinogenesis. Mod Pathol 2002;15:1087-1095.

28 Yeh TS, Tseng JH, Chen TC, et al. Characterization of intrahepatic cholangiocarcinoma of the intraductal growth-type and its precursor lesions. Hepatology 2005;42:657-664.
29 Shibahara H, Tamada S, Goto M, et al. Pathologic features of mucin-producing bile duct tumors: two histopathologic categories as counterparts of pancreatic intraductal papillary-mucinous neoplasms. Am J Surg Pathol 2004;28:327-338.

30 Zen Y, Fujii T, Itatsu K, et al. Biliary cystic tumors with bile duct communication: a cystic variant of intraductal papillary neoplasm of the bile duct. Mod Pathol 2006;19:1243-1254. 